\title{
Variations
}

Variations Revue internationale de théorie critique

\section{Le Parti de Lukács et la praxis sociale}

\section{Richard Westerman}

Traducteur : David Buxton

\section{CpenEdition}

\section{Journals}

Édition électronique

URL : https://journals.openedition.org/variations/1989

DOI : 10.4000/variations.1989

ISSN : 1968-3960

Éditeur

Les amis de Variations

\section{Référence électronique}

Richard Westerman, "Le Parti de Lukács et la praxis sociale », Variations [En ligne], 24 | 2021, mis en ligne le 03 juillet 2021, consulté le 07 juillet 2021. URL : http://journals.openedition.org/variations/ 1989 ; DOI : https://doi.org/10.4000/variations.1989

Ce document a été généré automatiquement le 7 juillet 2021.

Les ami•e•s de Variations 


\title{
Le Parti de Lukács et la praxis sociale
}

\author{
Richard Westerman
}

Traduction : David Buxton

1 Les textes fondateurs de la Théorie critique, Histoire et Conscience de classe de Lukács et Marxisme et philosophie de Karl Korsch, étaient le produit d'une crise dans le marxisme européen. Publiés tous les deux en 1923, ils représentaient une réponse aux révolutions réussies et échouées : alors que les bolchéviques avaient pris le contrôle de la Russie en dépit de son sous-développement, les gouvernements communistes en Hongrie et en Allemagne avaient été rapidement renversés en raison d'un manque de soutien populaire. Fait notable, Lukács et Korsch avaient fait partie de ces gouvernements Lukacs en première ligne avec l'Armée rouge hongroise. Bien que mémorablement dénoncées comme «un marxisme des professeurs» par l'orthodoxie soviétique naissante, les lectures profondément philosophiques de Marx développées par Lukács et Korsch dérivent bien de leur implication personnelle dans des situations révolutionnaires pratiques.

2 Le fait que ces livres ont été écrits, comme l'observe Lukacs, « comme des tentatives pour clarifier, à l'usage de l'auteur lui-même et de ses lecteurs, des questions théoriques du mouvement révolutionnaire $»^{1}$, est généralement oublié. C'est notamment évident dans la réception du concept de réification. Schématiquement, la réification décrit une pathologie sociale où des individus comprennent la société et les relations sociales à travers des lois fixes et immuables, avec pour conséquence qu'ils se sentent isolés et dans l'impossibilité de changer la société. On suppose généralement à tort - que la solution de Lukács est une version modernisée de l'idéalisme allemand, selon lequel le prolétariat prend conscience soudainement qu'il est le créateur de ce monde objectif, et ainsi se réapproprie spontanément sa création pour s'émanciper. En conséquence, l'argument de Lukács sur le rôle du parti dans l'essai ultime d'Histoire et Conscience de classe est lu au prisme de cette mésinterprétation; on l'accuse d'ouvrir la voie à un État centralisé sous le contrôle d'un parti autoritaire. Selon cette interprétation répandue, Lukács croit apparemment que parce que le prolétariat 
n'avait pas pris conscience qu'il est le sujet de l'Histoire, le parti révolutionnaire a simplement dû agir à sa place. On voit en Lukács le partisan d'un parti blanquiste qui devait se dégrader en dictature postrévolutionnaire.

Étonnamment peu des interprètes de Lukács ont reconnu qu'en réalité il envisage un parti beaucoup plus démocratique. La raison principale de cette mésinterprétation répandue est l'incapacité à comprendre correctement ce que Lukács veut dire par son concept-clé de réification, et par la façon dont ce concept influence sa théorie de l'organisation du parti. La plupart des exégèses de Lukács croient que la réification est une erreur commise par un sujet pensant - même si l'erreur s'explique par des facteurs sociaux. Le parti devait alors essayer de corriger l'erreur. La réification ne décrit pas, cependant, une épistémologie, mais d'emblée une forme de praxis. Le parti de Lukács n'est pas là pour jouer le rôle d'un leader sage qui guiderait le prolétariat - il est là pour fournir un point de fixation pour une praxis réellement déréifiée, et donc déréifiante. Plutôt qu'une structure blanquiste de révolutionnaires professionnels, le parti de Lukács est essentiellement une version plus institutionnalisée de la forme d'organisation préconisée dans Grève de masse, parti et syndicat de Rosa Luxemberg. Je vais commencer en retraçant le problème que Lukács essaie de prendre à la racine : la critique de Marx de la distinction entre l'État et la société civile dans Sur la question juive. Je veux montrer comment ce problème ne pouvait clairement pas être résolu par un parti d'avant-garde. Ensuite je me pencherai sur la position de Lukács ; j'affirmerai que sa vision du parti se positionne quelque part entre Lénine et Luxemberg en ce qu'il voit l'organisation formelle fournie par le parti comme essentielle pour une conscience de classe prolétaire réelle. Enfin, j'avancerai quelques voies qui pourraient offrir un modèle pour la sorte d'activité démocratique susceptible d'être un contrepoids aux structures sociales et politiques existantes.

5 Sur la question juive, texte écrit en réponse au pamphlet de Bruno Bauer sur la question d'une pleine émancipation juive au sein de l'État allemand, réinterprète radicalement le sens de la liberté sociale. Affirmant que la sécularisation de l'État ne mènerait qu'à la reproduction des divisions religieuses au niveau de la société, Marx met en question la division hégélienne de l'État et la société civile. Cette dernière, pour Hegel, est le domaine de la satisfaction particulière et de l'unité sociale immédiate; l'individu est relié à d'autres individus à travers un système économique de besoins, rationalisé par des institutions sociales construites sur cette nécessité de base. Par contraste, l'État est le domaine de la liberté rationnelle, où les citoyens sont unifiés en tant qu'individus rationnels universels. Pour Marx, il s'agit d'une forme aliénée de la liberté : d'abord, elle implique que des formes politiques semblent venir d'une force de raison universelle impersonnelle, et non de l'action humaine libre; ensuite, elle traite les catégories d'existence sociale comme invariables, nécessaires, ouvertes seulement au savoir, et non au changement. Marx propose, donc, de faire descendre le ciel à la terre, et de faire de la société un domaine de liberté en transformant les relations sociales elles-mêmes. La liberté réelle veut dire le contrôle collectif sur ces relations.

C'est ce type de liberté que Lukács voit en l'activité du parti. Mais je pense qu'il est immédiatement évident pourquoi un parti qui cherche à faire la révolution au nom du prolétariat ne serait pas en mesure de la réaliser. Un tel parti réduirait la classe ouvrière au rôle de spectateurs, aussi peu libres qu'avant. En fait, Lukács est très clair dans son rejet d'un parti vertical, et il est difficile de voir comment une lecture honnête et rigoureuse pourrait aboutir à une autre conclusion. Il dit explicitement que 
"théoriquement aussi, le parti communiste n'agit pas à la place du prolétariat » ${ }^{2}$, de peur qu'il réduise les masses à " une position purement contemplative » qui mène à « une surestimation volontariste de l'importance active de l'individu (du chef) et une sous-estimation fataliste de l'importance de la classe (la masse) $»^{3}$. Et il utilise de façon répétée le mot "réification" pour mettre en garde contre la fixation sur une seule forme d'organisation, l'isolement de celle-ci de toute critique ou tout changement par la masse. Lukács n'aurait pas pu être plus clair: un parti vertical, protostalinien représenterait un retour au manque de liberté caractéristique de la société capitaliste.

7 Lukács puise lourdement dans Rosa Luxemberg, tactique peut-être étrange en 1922 quand le succès des bolchéviques semblait signifier une victoire nette pour l'idée léniniste d'une structure disciplinée de révolutionnaires. La grève de masse dans laquelle Luxemberg a investi ses espoirs devait susciter le développement spontané de la conscience de classe en obligeant toutes les strates de la classe ouvrière à s'autoorganiser. Le parti de Luxemberg jouerait un rôle très secondaire, à peine plus en fait qu'une sorte de secrétariat, et certainement pas de direction.

8 Néanmoins, Lukács loue Luxemberg de façon répétée pour sa perspicacité. Il soutient explicitement ses critiques des partis communistes occidentaux qui ont sous-estimé l'action de masse, pensant que seul un parti éduqué était prêt à diriger. Il affirme, cependant, qu'elle fait l'erreur opposée, la critiquant pour sa «sous-estimation du rôle du parti dans la révolution ${ }^{4}$. Comme nous l'avons vu, Lukács ne pense pas que ce rôle implique de «diriger» dans un sens conventionnel. Afin de comprendre ce qu'il veut dire, il faudra regarder de plus près sa définition de la réification.

9 La plupart des interprétations de Lukács comprennent la réification comme une erreur épistémologique. On pense que le problème identifié par lui est que les catégories utilisées pour interpréter la société capitaliste sont trop abstraites et formelles. En conséquence, on pense que son projet est de remplacer de telles catégories avec d'autres, plus substantielles, qui reflètent la réalité qualitative sous-jacente avec plus de justesse. Malheureusement, cette interprétation ne résiste pas à une lecture attentive du texte ${ }^{5}$. La réification - verdinglichung, « chosification » - ne renvoie pas à un problème d'abstraction, à une quantité opposée à un substrat qualitatif, mais plutôt à l'ossification non dialectique des formes en choses qu'on ne peut changer. Cela est assez clair dans l'essai central du livre, "Réification et la conscience du prolétariat». Là, Lukács présente une interprétation de ce qu'il appelle la " philosophie bourgeoise ", la pensée allemande classique de Kant, Fichte et Hegel. Il identifie la préoccupation épistémologique de cette tradition: elle commence avec la séparation du sujet et de l'objet. Donc, sa question centrale est : dans quelle mesure notre connaissance et ses formes peuvent-elles correspondre à une réalité qui est externe à la conscience ? Ce point de vue épistémologique, affirme Lukács, nous réduit à être de simples spectateurs de la société, pensant qu'on ne peut saisir celle-ci qu'à travers des formes prédéterminées. Le problème, selon Lukács, n'est pas que ces formes soient fausses ; plutôt, c'est la tentative même de séparer sujet, objet et conscience, les uns des autres. On peut voir avec plus de détails ce que Lukács veut dire par « réification » dans la façon dont il parle du parti.

10 En premier lieu, le parti de Lukács sert essentiellement de forme institutionnelle de la conscience de classe des prolétaires. Sans parti, cette conscience serait sans forme, immédiate ; le prolétariat doit se donner une forme institutionnelle à sa conscience de soi, afin de se comprendre correctement. Le parti est donc la forme que le prolétariat 
révolutionnaire se donne à lui-même. Les sections les plus avancées de la classe ouvrière s'organisent en parti. Comme le dit Lukács : «L'autonomie organisationnelle du parti communiste est nécessaire pour que le prolétariat puisse apercevoir immédiatement sa propre conscience de classe comme figure historique ; [...] pour que toute la classe élève à la conscience sa propre existence en tant que classe ${ }^{6}$. Tandis qu'un parti blanquiste serait là pour dire aux ouvriers ce qu'il faut penser, le parti de Lukács incarne le prolétariat dans ses formes organisationnelles. De plus, ces forces ne sont pas qu'une représentation de ce qui existe déjà - une représentation plus ou moins juste d'un substrat sous-jacent du travail ou de l'essence. Au lieu de cela, Lukács dit que le parti est « l'acte de devenir conscient de soi » du prolétariat. Ce n'est qu'en adoptant une forme pour lui-même que le prolétariat devient vraiment une classe.

11 De plus encore, les liens intimes qu'établit Lukács entre forme et existence indiquent comment la réification pourrait revenir comme un problème dans l'organisation du parti. Bien que des questions de tactique jouent un rôle dans l'organisation, celles-ci ne devraient pas mener à l'imposition de certaines formes au nom de l'exigence. Plutôt, ce qui est décisif, c'est que ces formes proviennent de l'auto-organisation du prolétariat: « ...la formation ne peut être que l'œuvre consciemment accomplie des ouvriers ayant une conscience de classe ${ }^{7}$.» En conséquence, l'organisation ne consiste pas en une action une fois pour toutes et pour de bon. Lukacs n'essaie pas de remplacer une série de formes (abstraites, quantifiables, capitalistes) avec d'autres, plus "authentiques", " qualitatives ». Faire cela serait, dit-il, courir le risque du retour de la réification, qu'il identifie avec les structures organisationnelles de la direction du parti. Pour Lukács, ce n'est pas tant ce que le parti fait qui compte, mais plutôt les occasions qu'il offre aux prolétaires de s'impliquer activement en dessinant les formes de leur existence: «Ce n'est qu'en devenant pour chacun de ses membres un monde de l'activité que le parti communiste peut dépasser réellement le rôle de spectateur de l'homme bourgeois... ${ }^{8}$ "

12 Lukács identifie le parti comme le dépassement pratique de la réification: "L'organisation est la forme de la médiation entre la théorie et la pratique ${ }^{9}$. Comme Luxemberg, il rejette un parti blanquiste qui prend le contrôle au nom des ouvriers. Mais il va au-delà de Luxemberg en insistant sur une forme institutionnelle fluide pour la conscience prolétaire, sans quoi celle-ci serait vague et sans effet. La déréification donc est nécessairement pratique - elle implique un engagement délibéré dans des pratiques qui donnent de la forme à son existence. Le parti est la conscience pratique, l'incarnation de ses formes de façon à permettre leur transformation.

Bien que l'argument de Lukács s'appuie spécifiquement sur les conditions des classes ouvrières industrielles et la construction phénoménologique d'une conscience de soi prolétaire, je pense que son concept fondamental de praxis déréifiée peut s'appliquer plus généralement à une organisation démocratique et progressiste. Même au sein des formes sociales et politiques actuelles, on peut utiliser l'idée de réification pour critiquer les discours universalistes des droits ancrés dans un point de vue fixe ; ceux-ci rendent impossible la négociation des frontières de la citoyenneté ou de l'appartenance à un groupe d'une manière substantielle. Plus radicalement, cependant, le parti de Lukács nous offre un modèle pour l'action sociale de grande envergure.

La démocratisation, pour Lukács, entraînerait, de façon beaucoup plus compréhensive, l'implication dans la formation de nos relations sociales, et non simplement la réforme des catégories légales et politiques. Nous devrions comprendre les formes sociales au travers de l'idée des pratiques - c'est-à-dire, des interactions structurées, répétables, 
qui acquièrent un sens dans la totalité d'une culture. Ce sont ces pratiques qui deviennent réifiées. Plutôt que de les voir comme des choses que nous faisons, des choses qui sont rechargées de sens uniquement parce que nous continuons à les pratiquer, nous les traitons à tort comme fixes et immuables. Les pratiques sociales peuvent sembler presque divinement sanctionnées. Alternativement, on pourrait inventer une théorie supposément scientifique qui explique ces pratiques en termes de nature humaine éternelle, non modifiable, qui s'exprime inévitablement en formes sociales spécifiques. Nous ne pouvons agir que de cette façon, semble-t-il.

La déréification entraînerait une transformation délibérée de ces pratiques: nous devrions, dirait Lukács, traiter nos pratiques comme des choses qu'on peut adapter aux circonstances. Nous ne pouvons recréer des formes sociales à volonté à partir de rien mais, en même temps, en reconnaissant que les formes en tant que pratiques sont ce que nous faisons, nous pouvons les ouvrir à la transformation régulière. Suivant une suggestion de Sourayan Mookerjea, j'aimerais citer le mouvement altermondialiste comme modèle. Les altermondialistes accueillent positivement la croissance des interactions mondiales et de la coopération engendrée par le mode de développement actuel. Ils rejettent, cependant, la pensée néolibérale disant que celui-ci ne peut se passer que d'une seule façon, déterminée par des processus économiques scientifiquement connaissables. Le mouvement altermondialiste essaie donc de développer des pratiques sociales alternatives, s'orientant vers une redéfinition positive de l'interaction sociale, non le rejet irréfléchi de l'internationalisme.

Le modèle lukácsien du parti indique aussi comment cette activité pourrait être réalisée : un mouvement de la base et une orientation vers le problème de son autoorganisation. Autrement dit, les mouvements d'émancipation ne devraient pas s'orienter de façon instrumentale vers une finalité particulière; plutôt, ils doivent consacrer une grande part de leur énergie à eux-mêmes, à travailler sur la manière dont ils se tiennent ensemble. Ce faisant, ils offrent à leur membres l'occasion pour la sorte de praxis déréifiée visée par Lukács.

Résumons: la compréhension lukacsienne du parti révolutionnaire vise à réaliser certaines des fins émancipatrices énoncées dans Sur la question juive. Plutôt qu'une structure centralisée composée d'avant-gardistes professionnels, le parti de Lukács est façonné par les aspirations de Rosa Luxemberg d'une auto-organisation de la base. Interprétant le parti comme la forme consciente des relations sociales, Lukács indique l'importance d'une forme de présentation objective de nos pratiques, si on veut comprendre notre existence sociale correctement. Mais il suggère aussi une nouvelle définition de la praxis. L'acte même d'auto-organisation, de modification consciente des pratiques qui composent notre totalité sociale et culturelle, est pour Lukács l'essence de la praxis révolutionnaire. Si nous acceptons certaines façons d'interagir comme éternelles et immuables, nous tombons dans la réification. C'est seulement en luttant continuellement contre l'ossification de nos pratiques en formes invariables que nous pouvons espérer être émancipés. 


\section{NOTES}

1. Georg Lukacs, Histoire et Conscience de classe (trad. Kostas Axelos et Jacqueline Bois), Minuit, 1960 , p. 15.

2. ibid., p. 400.

3. ibid., p. 390.

4. ibid., p. 339.

5. Voir par exemple, Andrew Arato et Paul Breines, The Young Lukacs and the Origins of Western Marxism, Seabury (New York), 1979.

6. Lukacs, p. 399.

7. ibid., p. 414.

8. ibid., p. 412.

9. ibid., p. 367.

\section{AUTEURS}

\section{RICHARD WESTERMAN}

Professeur de sociologie, University of Alberta (Canada). 